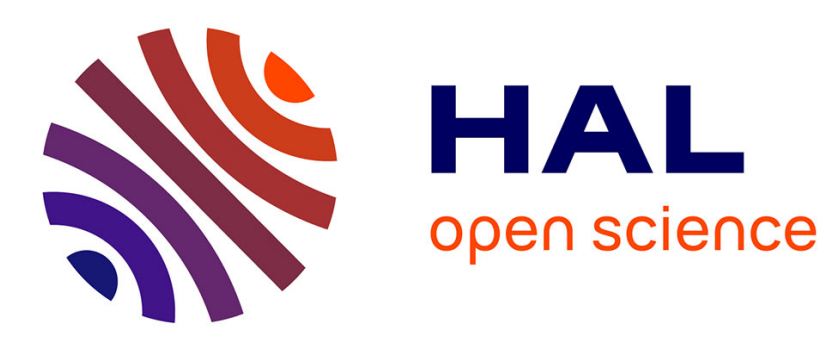

\title{
Psychodrame et démence : accompagnement de l'oubli sidératif par le corps et le geste
}

Mélanie Maurin, Guy Gimenez

\section{To cite this version:}

Mélanie Maurin, Guy Gimenez. Psychodrame et démence : accompagnement de l'oubli sidératif par le corps et le geste. Revue de psychothérapie Psychanalytique de Groupes, 2011, Mise en jeu et enjeu du corps, 57 (2), pp.143 - 143. 10.3917/rppg.057.0143 . hal-01393538

\section{HAL Id: hal-01393538 \\ https://hal-amu.archives-ouvertes.fr/hal-01393538}

Submitted on 7 Nov 2016

HAL is a multi-disciplinary open access archive for the deposit and dissemination of scientific research documents, whether they are published or not. The documents may come from teaching and research institutions in France or abroad, or from public or private research centers.
L'archive ouverte pluridisciplinaire HAL, est destinée au dépôt et à la diffusion de documents scientifiques de niveau recherche, publiés ou non, émanant des établissements d'enseignement et de recherche français ou étrangers, des laboratoires publics ou privés. 


\title{
PSYCHODRAME ET DÉMENCE : ACCOMPAGNEMENT DE L'OUBLI SIDÉRATIF PAR LE CORPS ET LE GESTE
}

\author{
MÉLANIE MAURIN \\ GUY GIMENEZ
}

Nous observons, au sein de nos institutions, une augmentation importante du nombre de patients présentant une démence. La démence, ou plutôt les démences telles qu'elles sont représentées dans le champ de la prise en charge du sujet âgé, renvoient essentiellement à une dimension de diminution, de perte et de manque avec au centre de la problématique : la dimension de l'atteinte mnésique. Au cours des rencontres cliniques, nous observons que les sujets déments évoquent le symptôme de l'oubli en premier lieu comme objet de leur souffrance. Les patients disent ne pas se souvenir, ne plus se rappeler. Les propos tels que «Je ne retiens plus rien », «Je perds la tête », « Je deviens fou ", reviennent régulièrement dans leur manière de se dire. Nous avons ainsi choisi de travailler plus spécifiquement cette dimension de l'oubli ; oubli que nous qualifions de sidératif. L'oubli sidératif émerge dans la surprise, crée un blanc, une rupture du lien. Le patient est comme sidéré, immobilisé dans ses possibilités à réaccéder à la trace qui semble perdue. Le retour à la relation se fait dans une rupture apparente d'avec le sens précédent, sans respect de la continuité conversationnelle, sans que le patient puisse en dire quelque chose. Tout se passe comme si l'expérience effractive de l'oubli elle-même venait à être gommée, annulée dans le vécu du patient.

L'objectif de notre travail est de repérer comment il est possible de réaccéder à des traces psychiques, en apparence inaccessibles, en passant par le corps, l'affect et la motricité. Nous faisons l'hypothèse

Mélanie Maurin, membre du Laboratoire LPCP, université de Provence ; Résidence Le Gauguin, bât. D, 28 chemin de Brunet, 13100 Aix-en-Provence ; maurin_melanie@yahoo.fr Guy Gimenez, membre du Laboratoire LPCP, maître de conférences HDR en psychologie clinique, université de Provence (Aix-Marseille I) ; 29 avenue Robert-Schuman, 13621 Aix-enProvence cedex 1 ; guy-gimenez2@wanadoo.fr 
qu'au-delà des expressions de l'oubli démentiel, que nous qualifions de sidératif, des fonctions psychiques du patient demeurent mobilisables, en vue de permettre une relance du processus de métabolisation. Pour mettre au travail nos hypothèses, nous utilisons le dispositif de psychodrame psychanalytique de groupe. Dispositif que nous aménageons en fonction des spécificités de cette population, notamment en termes de techniques d'intervention. Nous pensons que le psychodrame peut répondre aux nécessités de prise en charge des sujets déments, et ce autour de quatre grands axes.

Premièrement, nous observons que les patients déments ont parfois du mal à se dire verbalement. Les phénomènes démentiels conduisent à des manques de mots, utilisation d'un mot à la place d'un autre, perte du langage. L'interrelation passe alors d'avantage au travers de la dynamique émotionnelle et corporelle. Un des intérêts du psychodrame est que le jeu ne fait pas qu'utiliser le langage verbal. Il met en mouvement les corps, celui du patient, ceux des acteurs. Le passage par l'acte permet alors d'accéder à nombre d'éléments psychiques qui n'entrent pas dans le système des représentations verbales.

Deuxièmement, dans la démence les symptômes tels que l'agitation et la désorientation, provoquent des vécus d'angoisse important pour les patients. Le psychodrame, du fait de son dispositif groupal, permet un effet de contenance. Cette contenance groupale permet un apaisement des vécus d'angoisse dans l'expérience de tenir tous ensemble dans un même espace. Le groupe propose une enveloppe capable de recevoir et de contenir les mouvements émotionnels non élaborés. Il contribue à la constitution d'une enveloppe psychique que chacun peut intérioriser à la place d'un «Moi-peau » (Anzieu, 1985) trop rigide, trop « troué » ou trop inconsistant.

Troisièmement, les sujets déments se trouvent plus ou moins atteints dans leurs corps. Le vieillissement, qu'il soit qualifié de normal ou de pathologique, conduit à des pertes, des ralentissements, voire des incapacités physiques. Difficultés qui se trouvent grandement majorées par la démence. Les patients semblent perdre contact avec une partie de leur corporéité. Le psychodrame sollicite la dimension corporelle et sensorielle. Le passage par l'action permet une relance de ces dimensions, et un réinvestissement progressif de la corporéité.

Quatrièmement, le psychodrame favorise l'expression et la transformation d'expériences anciennes. Le passage par le corps et la gestualité permet d'accéder à des traces psychiques en apparence perdues. Nous faisons l'hypothèse qu'une trace en apparence perdue, au niveau verbal, reste disponible à autre niveau. Le psychodrame permet l'accès à des niveaux de représentation archaïque et primaire, et relance une démarche de symbolisation. Il y a ainsi possibilité de retrouver des inscriptions psychiques, et de favoriser une démarche du souvenir.

La tâche du clinicien en séance de psychodrame est complexe. Il est porteur des associations groupales, c'est à lui qu'incombe le travail 
de liaison des éléments restés jusqu'ici épars. Il assure une fonction de liaison, à l'interface des éléments individuels et groupaux. Nous observons que les spécificités des symptomatologies démentielles nécessitent un appui important, et un double positionnement : capacité d'attention flottante pour entendre la dimension inconsciente, et en même temps, présence soutenue et active dans l'ici et maintenant afin d'être à même de relancer et transformer les productions groupales. Ce double positionnement place le clinicien dans une situation paradoxale, qui lui demande un réajustement constant, comme une oscillation permanente entre deux pôles antagonistes et complémentaires. Pour ce faire, le thérapeute met au service du groupe son appareil à penser. Il y a ainsi un « penser » qui engendre les pensées, et un autre «penser » qui consiste à employer les pensées épistémologiquement préexistantes. Pour que les deux fonctionnent, il faut un appareil à penser les pensées (Bion, 1967).

\section{LE GROUPE ARC-EN-CIEL}

Le groupe arc-en-ciel a été mis en place au sein d'un établissement pour personnes âgées dépendantes. Il fonctionne depuis le mois d'octobre 2010, et il est animé en cothérapie par la psychologue et l'animatrice de l'institution. Il s'agit d'un groupe ouvert à tous les patients de l'établissement. Il est constitué régulièrement d'une dizaine de patients qui présentent des troubles démentiels, à symptomatologie plus ou moins avancée. Nous avons choisi de présenter la deuxième séance du quatrième mois de fonctionnement du groupe. Ce jour-là, neuf patients participent à la séance. Pour une meilleure lisibilité de notre texte, nous avons pris le parti de décomposer cette séance en trois parties. Pour chacune d'entre elles, nous allons présenter les éléments de la dynamique groupale, puis des axes d'élaboration. Au sein des éléments de la dynamique groupale, nous présentons les données sensorielles de la séance (ce qui est dit, vu, agi), ainsi que les mouvements internes qui sont les nôtres (ce que cela nous donne à penser, ressentir, associer - en italique).

\section{Partie 1 - Tension}

La mise en groupe commence par l'exclamation de Claude : «Tiens, il y a un homme aujourd'hui, alors on est sauvé ! » Des rires éclatent puis un long silence s'ensuit. Des regards s'échangent, la plupart portés vers Léon, assis à notre gauche, qui touche à plusieurs reprises son appareil auditif. Puis un silence.

La clinicienne prend un temps pour observer le groupe. Juliette a quitté l'espace de parole, elle déambule avec son fauteuil roulant, et effectue des va-et-vient à la frontière entre espace de jeu et espace de parole. Habituellement, Juliette se montre particulièrement attentive et 
immobile en séance. Un signifiant formel (Anzieu, 1987) vient à l'esprit de la psychologue : "Un corps bouge, un corps qui ne peut rester en place. »

Silence à nouveau. Un rappel des règles est effectué, à savoir les règles de libre association, de confidentialité et du faire comme si. Léon, assis à notre gauche, nous regarde avec attention. À la règle de libre association, il réagit en souriant : «Ah bon ? On peut tout dire... même ce qui fait pas plaisir ? »Quelques rires à nouveau dans le groupe, mais plus retenus, comme étouffés. Les regards deviennent fuyants, excepté celui de Line qui observe fixement Léonore assise près d'elle. Léonore qui soulève et abaisse de manière répétitive les cale-pieds de son fauteuil roulant. Nous associons alors le mouvement répétitif de Line, avec les allées et venues de Juliette. Nous pensons que quelque chose de difficilement pensable circule dans le groupe, et qu'il vient à s'exprimer dans ce mouvement répétitif.

Un silence s'installe. Juliette et Line poursuivent leurs va-et-vient. Le reste du groupe est particulièrement immobile. La psychologue écoute ce qui se passe à l'intérieur de son corps : une gêne dans la gorge. Elle associe à une montée de la tension psychique.

Elle observe à nouveau le groupe. Aimée, le visage tendu, regarde fixement Juliette qui continue ses va-et-vient : «Elle a pas fini, celle-là ? », dit-elle d'un ton fort. Suzanne reprend : «Elle serait mieux dehors ! » Juliette ne réagit pas, et continue ses va-et-vient. Les interventions d'Aimée, puis de Suzanne, provoquent un effet de violence. La psychologue pense que l'hostilité du groupe vient ici à s'exprimer, et qu'il tente de s'en débarrasser. Elle se dit qu'il lui faut dire au groupe quelque chose à propos de ce qui se joue dans l'ici et maintenant de la séance.

Un silence suit, puis la clinicienne énonce : «Mettre au dehors quelque chose de gênant... » Un long silence s'installe. Au bout de quelques instants, Juliette s'immobilise et regarde attentivement le groupe. La psychologue a l'impression que Juliette prend conscience de la présence du groupe seulement à cet instant, comme si le groupe venait de lui apparaître.

La clinicienne lui fait signe de les rejoindre dans l'espace de parole. Juliette avance vers l'espace de parole en souriant, elle attrape la main de la psychologue et dit: "C'est pour le machin que vous faites ça... Moi, je vais devoir aller chercher mes petits. » Sur le moment, la clinicienne ne perçoit pas de lien entre le discours de Juliette et ce qui $s$ 'agit dans la dynamique groupale. Dans l'après-coup, elle associe avec le geste qui tente d'aller chercher Juliette là où elle est. Et tenir la main comme on pourrait le faire avec un enfant.

La thérapeute décale sa chaise vers la gauche et accompagne le déplacement de Juliette jusqu'à ses côtés. Juliette prend alors place dans l'espace de parole, lâche sa main, souriante, mais son regard paraît triste. 
Ce premier temps du groupe est marqué par une forte tension psychique. Avec l'arrivée de Léon, des éléments latents sont réactivés, et s'expriment sur la scène groupale au travers notamment d'un fort vécu émotionnel. Le phénomène d'illusion groupale ne tient plus, le groupe perd ses repères, l'angoisse se diffuse et circule entre les participants. Nous repérons cette anxiété dans les manifestations comportementales du groupe, mais aussi à travers notre propre vécu interne en termes de montée de tension psychique. Les mouvements moteurs répétitifs de Juliette et Léonore viennent exprimer une décharge de ce trop plein. Trop plein d'excitation, d'hostilité et tentative de décharge groupale portées par les deux patientes. Avec un même signifiant : s'éloigner, se rapprocher. Les regards du groupe qui deviennent fuyants évoquent une perte d'accroche. La fonction de maintenance groupale semble inefficiente. Ce vécu commun apparaît en tant que déstabilisation du système groupal, un changement de structure, changement catastrophique au sens de Bion. L'élément nouveau qui émerge dans le groupe renferme une forme potentiellement disruptrice, qui fait violence à la structure du champ groupal. Ce changement est vécu en terme de perte, l'enveloppe groupale est remise en cause. Moment éprouvé par le groupe en termes de désorganisation et de souffrance.

La tension trouve son point culminant lors de la réaction de Léon à la règle de libre association : tout peut être dit, même l'agressivité, l'hostilité, la destructivité. Léon semble porter cette dimension le groupe (fonction phorique décrite par René Kaës [1994]). C'est à pour ce moment-là que Juliette émerge comme mauvais objet à exclure. Le négatif du groupe trouve ici à se fixer. Juliette, en tant que «porte-négatif », et qu'il faut mettre à distance pour sauvegarder le reste du groupe. Notre intervention cherche à ramener Juliette dans l'enveloppe groupale. Nous choisissons de passer par une communication gestuelle, et non verbale, afin d'être au plus près de ce qui se joue dans le groupe. Nous signifions ainsi une alliance avec Juliette, porteuse du négatif, et prenons la responsabilité de l'agressivité groupale. Il y a nécessité de restaurer l'enveloppe groupale, relancer la fonction de contenance. En proposant à Juliette une place près de nous, nous souhaitons convoquer un éprouvé de peau commune qui viendrait comme un apaisement.

\section{Partie 2 - Ça brûle}

La psychologue annonce le premier temps de la séance. Un court silence dans le groupe, puis Aimée prend la parole : «J'ai vu ma maison brûler », annonce-t-elle. Un silence plus long s'installe. Aimée reprend : «Tout brûle... la grange... il y a les animaux dedans. Les vaches, les chèvres, les chevaux... tout brûle. »La psychologue est sensible au changement du ton de la voix d'Aimée, qui est plus ferme, ainsi qu'au changement de temps de son récit : du passé au présent. 
Des traces anciennes viennent se réactualiser dans l'ici et maintenant de la séance.

Tout en évoquant la scène, le regard d'Aimée reste au-devant d'elle dans le vide laissé au milieu de l'espace de parole. Les expressions de son visage sont marquées, ses yeux grands ouverts. La thérapeute a l'impression que la scène évoquée se présente à elle dans l'ici et maintenant de la séance. Elle fait l'hypothèse d'une reviviscence de l'expérience traumatique qui passerait notamment par un canal sensoriel : ici, le visuel.

Un long silence s'installe. Juliette, Lucie et Sophie regardent Aimée avec attention. Cette convergence des regards vers Aimée produit un effet qui apparaît contenant. Il semble que le groupe tente de tenir Aimée par un agrippement par le regard pour éviter une chute, un effondrement.

Léon signale à la clinicienne ne rien avoir compris ; elle reprend les éléments rapportés par Aimée en restant au plus près de son discours. Léonore, quant à elle, a repris l'action du lever/baisser des cale-pieds de son fauteuil, mais sur un rythme plus rapide. Line réagit vivement : « Arrête. » Léonore relance à son tour : «Oh, la ferme »d'un ton agacé.

La clinicienne ressent une montée de la tension psychique dans le groupe et en elle-même. Elle se trouve saisie par l'effet de violence et de surprise que provoque en elle ce "La ferme » de Léonore. Léonore habituellement toujours calme et ne communiquant quasiment pas verbalement. Quelque chose trouve à nouveau décharge d'un trop plein. Décharge assurée, depuis le début de la séance, par deux participantes dont les symptomatologies sont les plus avancées. La psychologue pense qu'il y a de l'archaïque qui s'exprime ici. Ce n'est que dans l'après-coup que nous entendons le lien entre l'énoncé de Line et ce qui était alors évoqué dans le groupe : la ferme qui brûle.

Aimée reprend son discours mais articule de moins en moins ses propos, ce qui les rend, par moments, quasiment incompréhensibles. Son corps commence à s'affaisser progressivement du côté droit. Après : "Un corps bouge, un corps qui ne peut rester en place », c'est un nouveau signifiant formel qui vient à l'esprit de la thérapeute : "Un corps qui tombe, qui glisse. » Elle prend alors conscience de l'attention soutenue portée à Aimée depuis plusieurs minutes.

La psychologue observe à nouveau le groupe. Cette fois-ci, tous les regards sont dirigés vers Aimée. La psychologue a la sensation d'un arrêt sur image, d'une mise en suspens. Elle pense : le temps s'est comme arrêté, et associe avec la modification temporelle du discours d'Aimée précédemment : du passé au présent.

Après un silence, Aimée regarde le groupe, et se redresse légèrement. Elle poursuit son discours, et donne des précisions sur l'événement. Progressivement, son discours devient décousu, en apparence illogique. Le ton de sa voix est irrégulier, sa bouche se tord. Les mani- 
festations de son hémiplégie droite reviennent en première ligne, son corps est particulièrement déséquilibré. La psychologue est sensible à ce qu'elle observe du corps de la patiente. Elle éprouve des difficultés à faire des liens dans son discours, les énoncés apparaissent hétérogènes, sans logique lisible.

Lucie et Léon manifestent alors ne pas avoir entendu. La clinicienne pense : «Ce qui n'est pas entendu, ce qui ne s'inscrit pas. » Elle associe à la fonction d'inscription des traces psychiques.

Elle répète alors tant bien que mal les propos tenus par Aimée au groupe. Un long silence s'installe. Chacun semble en contact avec un univers qui lui appartient. La clinicienne porte son regard, qu'elle pense enveloppant, tour à tour sur chacun des participants sans s'y arrêter vraiment. Elle cherche à représenter une continuité au travers de ce regard, afin de restaurer l'enveloppe du groupe, et à faire éprouver à chacun une boucle contenante de la vision.

Son regard s'arrête alors sur Suzanne. Les yeux rouges, les joues empourprées, elle tient fermement des deux mains un petit sac posé sur ses genoux. Le regard posé au devant elle, comme dans le rien. Elle apparaît particulièrement émue.

La thérapeute reprend son regard groupal, puis énonce : «Des événements apportés dans le groupe qui génèrent des émotions. » Un silence à nouveau, puis Suzanne intervient : «Oui, c'est difficile de perdre sa maison... moi, ça fait trois ans que je suis ici. » Son visage s'empourpre, des larmes roulent sur ses joues. Des échanges s'ensuivent. Claude, Sophie et Aimée évoquent leurs entrées respectives dans l'établissement. Marie, après plusieurs hésitations, dit ne pas se rappeler. Un nouveau silence suit. La clinicienne reprend les énoncés et interpelle le groupe dans le sens d'une construction de scénario. Après un nouveau court silence, Lucie annonce très souriante : «Eh bien, moi, je pense à une maison... une maison familiale, pas un château. Une maison avec des gens qui s'y sentent bien. » La psychologue constate la rupture soudaine d'avec la tonalité de ce qui était dit précédemment, et pense à un mouvement défensif du groupe porté par Lucie.

Quelques échanges émergent qui viennent reprendre l'association de Lucie. Peu à peu, un scénario de jeu est décidé. Ce serait une maison, une maison accueillante où chacun pourrait se sentir chez soi. Il y aurait une propriétaire et des voisins qui viendraient la visiter. $L a$ psychologue repère la rapidité avec laquelle le scénario s'est construit et se demande s'il s'agit d'une fuite en avant du groupe.

Lucie se propose d'emblée pour jouer un des voisins qu'elle prénomme Lanvin. Léon, le second voisin qu'il qualifie de «pas content du tout » et prénommé Hubert. Claude est sollicitée par Aimée pour jouer la propriétaire, elle accepte et sera Julie.

L'association exprimée par Aimée apparaît comme une première figuration de ce qui se joue dans le groupe. Quelque chose est en train de brûler, nous entendons ici que c'est de la dimension de l'hostilité 
groupale dont il s'agit. Hostilité réactivée et repérée en terme de dangereux, qui provoque la disparition et peut être meurtrière. La convergence des regards, l'attention soutenue du groupe vers Aimée produit une orientation commune et unique. Le groupe semble se reconnaître dans son discours, ce qui convoque un mouvement de réunification. Quelque chose de l'enveloppe se restaure. Nous sommes à nouveau tous ensemble.

Léon, puis Lucie, disent ne pas comprendre, et expriment ainsi qu'une partie du groupe demeure inaccessible. Au-delà de leurs problématiques avérées de surdité, il y a comme une résistance à entendre, à recevoir. La fonction d'inscription du groupe se trouve en partie défaillante. Les difficultés que nous éprouvons à assurer la fonction que nous avons nommée " écho amplificateur » indique un effet de contamination. Au-delà de la prise en compte des problématiques de surdité, cette fonction est venue en tant qu'accompagnement des phénomènes d'oubli dans le groupe. Fréquemment, d'un échange à l'autre, les patients expriment leur sensation de perdre certaines traces groupales. Ces dernières sont parfois reprises par le groupe lui-même, assurant ainsi sa propre fonction de contenance et de maintenance. Mais il arrive aussi à d'autres moments de la vie du groupe, que ces traces ne soient pas reprises. Le groupe semble alors comme sidéré, plongé dans un vécu de perte qui signe pour nous d'un travail du négatif à l'œuvre. Nous avons alors à assurer cette fonction au nom du groupe : fonction d'écho, de mémoire, de gardien des traces groupales. Nous avons été conduits à l'élaboration d'une fonction spécifique qui fait partie intégrante du dispositif qui constituerait un soubassement du «porte-voix » (Pichon Rivière), ou de porte-parole de la mère (Kaës) permettant de rendre accessible et figurable des traces intrapsychiques.

Aimée semble progressivement perdre sa maintenance (Anzieu, 1995), figurant ainsi un vécu d'effondrement psychique. L'émotion groupale va être portée par Suzanne, dans ce qui pourrait être qualifié d'une émotion dépressive. L'économie libidinale du groupe y trouve un intérêt. Au travers des larmes de Suzanne, c'est une dimension dépressive groupale qui peut s'exprimer, se partager, être mise au dehors. Nous pensons que le groupe évolue, qu'il est en contact avec des éléments plus élaborés. Nous faisons le lien avec la position dépressive telle que Melanie Klein l'a conceptualisée. Avec ces éléments dépressifs, vient une possibilité de temporalité. Alors que, dans le discours d'Aimée, passé et présent semblent être confondus. Émerge ici une première différenciation entre hier et aujourd'hui, et la possibilité pour le groupe de se remémorer en se reconnectant à des inscriptions psychiques plus ou moins anciennes. Ces inscriptions évoquent la perte (ici, perdre sa maison) et convoquent la dimension du deuil.

La proposition de Lucie apparaît alors comme une réaction défensive. La maison qui brûlait devient une maison brusquement accueillante. Le feu est éteint en apparence. L'adhésion immédiate du 
groupe signe d'un retour massif à une volonté de cohésivité adhésive et une tentative groupale pour se raccrocher à du même. Nous pensons à une forte résistance à traiter ce qui est vécu comme "dangereux », dans un mouvement de mise à distance de l'hostile dans un scénario retourné en son contraire.

\section{Partie 3 - L'oubli}

Nous prenons un temps pour décider des différents lieux du jeu : la porte d'entrée, le couloir, le salon. La thérapeute se rend dans l'espace de jeu et se positionne, tour à tour, à trois endroits différents, et figure les actions : taper à la porte, l'ouvrir, avancer dans le couloir, prendre place dans le salon. Lucie regarde chacun des gestes avec beaucoup d'attention. La thérapeute repense aux séances précédentes, et aux oublis fréquents manifestés par Lucie en début de jeu. Elle pense que la patiente cherche par ce regard à inscrire chacun des éléments figurés.

Lorsque les participants sont dans l'espace de jeu, un rappel verbal des personnages est effectué. La clinicienne se positionne tour à tour derrière chacun des personnages, rappelle leurs noms et identités, et ajoute un toucher des épaules des deux mains. Lorsque cela vient à son tour, Lucie répète à plusieurs reprises : «Oui c'est ça, je suis Lanvin. » Sa voix est particulièrement aiguë. Lucie apparaît anxieuse, comme dans l'inquiétude d'un oubli qui pourrait venir dans le jeu.

Le jeu commence, la propriétaire est assise dans le salon, Hubert et Lanvin sont devant la porte d'entrée. La psychologue est restée debout près de l'espace de jeu. Hubert tape à la porte et entre. Il salue la propriétaire, s'assoit et avance d'emblée être le voisin pas content. La propriétaire surprise le questionne. Elle aurait construit un mur non réglementaire sur son terrain. La propriétaire hésite. Un silence s'installe. Lanvin tape alors à la porte et entre dans le salon. Il salue chacun des personnages d'une voix particulièrement sonore : "Bonjour madame, bonjour monsieur. » Une fois les personnages salués, Lanvin se positionne un peu en retrait tout en restant debout.

Après une courte pause, Lanvin énonce : «À vrai dire, je ne sais pas trop ce que je fais ici. » Un silence s'installe à nouveau. Les trois personnages en jeu se regardent tour à tour. Julie intervient : «Ce que vous faites ici... ce que vous faites ici, c'est à moi de vous dire ce que vous faites ici ? »La psychologue a la sensation que Julie ne sait plus elle-même la raison de la visite de Lanvin.

Après un nouveau silence, Lanvin reprend soudain très souriant : «Faut dire que je perds un peu la tête, j'ai des problèmes de mémoire et je me souviens plus très bien. » Rires de Lanvin et de Julie. Hubert touche son appareil auditif, et effectue un geste de la tête qui semble signifier qu'il n'a pas compris. La clinicienne associe intérieurement à "quelque chose qui n'est pas entendu, qui n'est pas audible». 
Un silence suit, dans lequel les personnages sont particulièrement immobiles. Lanvin ne sourit plus, son visage est spécialement grave. Hubert regarde au sol, et Julie fixe droit devant elle un point que je ne parviens pas à repérer. La psychologue associe au moment de mise en suspens précédent : à nouveau, le temps semble s'être arrêté.

Lanvin à nouveau souriant : «Alors, si vous non plus vous ne savez pas, je suis perdu. » Un nouveau silence s'installe. La psychologue pense qu'il est nécessaire d'intervenir pour relancer l'accès aux traces et ainsi au souvenir. Au moment même où la psychologue va intervenir, elle ressent une forte hésitation, se demandant intérieurement qui est qui et qui fait quoi dans ce jeu.

La clinicienne regarde les participants restés dans l'espace de parole. Line a repris ses mouvements répétitifs, Juliette a les yeux fermés, Aimée s'est écroulée sur son fauteuil. Elle cherche le regard d'Angélique, cothérapeute, afin d'y trouver une aide dans la démarche de remémoration. Mais Angélique, le regard troublé, lui fait signe de ne plus être sûre non plus de l'identité de chacun. Écoutant ce qui se passe en elle, la psychologue a une impression de chute, de vide, sans accroche, ni personne sur qui s'étayer. Elle tente d'appeler son superviseur interne (Casement, 1985), qui reste désespérément silencieux.

Lanvin se met alors à parler beaucoup, plaisante sur ses « trous de mémoire », et rit à plusieurs reprises. La clinicienne a la sensation que Lanvin tente de remplir le vide par un flot sonore. D'un trop vide, elle passe ainsi à une soudaine impression de trop plein.

Léon intervient : «Et le mur ? On fait quoi pour le mur ? » Les souvenirs des éléments du jeu reviennent à l'esprit de la psychologue soudain. Elle pense que Léon joue à nouveau une place particulière dans le groupe.

Julie et Lanvin hésitent toujours. La thérapeute décide alors d'entrer dans le jeu afin d'intervenir au sein même de l'espace intermédiaire par le «faire comme si ». Elle se présente comme étant la sœur de la propriétaire venue lui rendre visite. À son entrée en jeu, elle restitue les identités de chacun en les saluant.

Le jeu se déroulera ensuite sans nouvelle manifestation d'oubli. Hubert tiendra sa position de manière autoritaire et demandera à Julie de démolir le mur qui n'est pas réglementaire. Lanvin tentera à plusieurs reprises de médiatiser la situation sans succès. Il n'y aura pas de compromis à l'amiable possible. Le jeu se termine sur une impossibilité à trouver une entente.

Dans le temps d'après-jeu, peu d'échanges suivront : le groupe demeure silencieux malgré nos invitations à dire. Devant ce vide, nous énonçons quelques éléments. "Cette maison n'était peut-être pas si accueillante que cela. » M. Léon ne bougera pas du positionnement de son personnage et parlera en son propre nom : «C'est inadmissible de construire des murs comme cela, moi je ne suis pas d'accord », dira-t-il à plusieurs reprises d'un ton agacé. Aimée reprendra : «Il 
était méchant le voisin. » Dans l'après-coup, nous faisons le lien avec son discours lors du premier temps de la séance : c'est effectivement le « voisin méchant» qui a mis le feu à la ferme. Il s'agit d'une intervention «double face » (Gimenez, 1996, 2003) qui concerne à la fois une inscription individuelle d'Aimée, et figure ce qui se passe dans la dynamique groupale.

Un retour sur les fonctions évoquées. La fonction «mise en scène » apparaît comme une volonté d'amorçage de l'action et s'inscrit dans notre volonté d'accompagnement de l'oubli démentiel. Nous avons observé que le début du jeu peut être vécu par les patients déments comme particulièrement anxiogènes. Les manifestations de l'oubli émergent souvent à ce moment-là avec une confusion des lieux et des personnages. Nous constatons que l'utilisation de notre propre corporéité dans cet espace avant le jeu, va dans le sens d'un amorçage. Des participants ont rapporté avoir pu jouer en se remémorant les actions que nous avions produites précédemment. Il y a là le passage par un appui visuel (dans un mouvement identificatoire) pour inscrire et faire appel aux traces.

Concernant le rappel des personnages, nous utilisons le toucher, et avons nommé cette fonction le «toucher renforcement ». Nous y voyons une tentative d'ancrage, un passage par le vécu corporel pour faire appel à des fonctions plus élaborées. Le choix des épaules se justifie en ce sens qu'il s'agit d'un endroit du corps qui est facilement contactable.

Dans cette séance, nous constatons que l'utilisation de ces deux fonctions successives ne semble pas suffisante pour le travail du souvenir. L'oubli émerge d'emblée dans le jeu porté par Lucie dans le personnage de Lanvin, qui porte, pour le groupe, cette dimension. Cela est dû en partie à l'importance de sa symptomatologie, mais également au fonctionnement groupal au sein duquel elle prend une place particulière que nous nommons porte-oubli. Ici, l'oubli ne va pas s'arrêter à la manifestation de Lucie puisqu'il va contaminer l'ensemble de l'espace groupal. Les hésitations que nous percevons à l'intérieur de notre psychisme signent une transmission de cet oubli. Nous faisons l'hypothèse d'un travail du négatif à l'œuvre à ce moment-là de la dynamique groupale : les liens sont attaqués. L'oubli circule et provoque de la sidération.

C'est le rappel de Léon concernant le mur qui nous permet une réaccessibilité aux traces avec précision, et une possibilité du souvenir. Le mur, élément qui n'avait pas été donné lors de la construction du jeu, et qui émerge comme étayage : objet solide contre lequel il est possible de s'adosser (être dos au mur, s'appuyer au mur). Nous faisons le lien avec la fonction de l'adossement et des fonctions psychiques qui s'acquièrent au travers de cette expérience. Le ressenti de la colonne vertébrale, se sentir debout et unifié : nos pensées ont de nouveau de quoi tenir debout (fonction de soutenance du moi peau). 
Nous décidons d'entrer dans le jeu en proposant un personnage du fait de la massivité des effets de sidération. Nous pensons qu'il nous faut être dans l'action et être à même d'intervenir directement si besoin en étant dans le champ de l'intermédiaire du faire comme si. Il n'y aura pas d'autres manifestations d'oublis au cours du jeu, mais des positionnements fermes et irrévocables, comme des accrochages massifs pour éviter la chute.

\section{Évolution du groupe}

L'évolution des séances nous amène à constater une évolution de la dynamique du groupe. Après un premier temps d'illusion groupale (Anzieu, 1971), où le groupe fonctionnait sur un mode de fusionnalité, des différences se font peu à peu jour entre les participants. Avec cette différenciation, émerge également une possible expression de mouvements hostiles restés latents. Les associations groupales émergent avec plus de facilité, les participants prennent la parole et les silences sont plus présents, comme tolérés par le groupe. Nous constatons également la mise en place de défenses, qui se rigidifient et qui se manifestent notamment par des accrochages à du concret. Il semble y avoir pour le groupe une inquiétude à aller vers du fantasmatique. Le travail de l'imaginaire devient ainsi par moment difficile, le clinicien est sollicité en lieu et place du rêve groupal. Les thématiques de jeu, d'une séance à l'autre, viennent progressivement constituer une chaîne associative autour du voyage. On est dans le dehors de l'institution, l'évasion, l'ailleurs comme possibilité de rêver un lieu lointain porteur des idéaux et fantasmes du groupe.

\section{Conclusion}

La pratique du psychodrame psychanalytique de groupe avec les patients déments nous conduit à repenser les modalités de fonctionnement de ce dispositif. Même lorsqu'il n'est pas en jeu, nous observons que le clinicien se doit d'être dans une mise en acte, nous dirions volontiers une mise en corps de la dynamique groupale. La corporéisation passe par le groupe et le clinicien : le patient peut alors s'y identifier. Nous constatons qu'il y a des possibilités d'étayages successifs de l'un à l'autre : des phénomènes de coétayage. Certaines fonctions psychiques viennent ainsi à être relancées par la dynamique groupale au travers d'un autre et de plus d'un autre.

Au-delà d'une étiologie organique, il y aurait donc une dimension psychique aux conditions et manifestations de l'oubli chez les patients déments. Selon les techniques thérapeutiques qu'il utilise, le clinicien peut favoriser une réémergence du souvenir. Pour cela, un amorçage est souvent nécessaire, amorçage qui va passer par d'autres canaux de la communication que la communication verbale seule : un geste, un 
signe, une expression faciale et corporelle, sont autant de marqueurs langagiers qui peuvent venir soutenir et relancer une dynamique de l'échange, marqueurs qu'il va falloir repérer et définir avec précision.

Nous repérons que les modalités d'accompagnement de l'oubli sidératif nécessitent un passage par le sensoriel, la corporéité, pour préfigurer une forme qui était déjà là auparavant mais qui semble être attaquée au point de disparaître psychiquement, comme effacée. Mise en corps qui demande au clinicien une adaptabilité et une certaine souplesse dans les techniques qu'il engage. Les interventions du clinicien en séance sont sous-tendues par sa capacité à recevoir et accueillir les éléments groupaux. Plus particulièrement les éléments non verbaux, exprimés par le groupe au travers de la dynamique émotionnelle. Un geste, un regard, une posture sont autant de marqueurs qui viennent parler de ce qui circule entre les participants du groupe. Ces éléments passent par l'appareil psychique du thérapeute, qui est alors à même de percevoir dans sa propre intériorité, les effets de la dynamique inconsciente. Lorsque ces éléments sont suffisamment gardés et élaborés par le clinicien, il décide de le restituer au groupe sous forme d'intervention agie dans le jeu, ou verbalisée dans l'espace de parole. Cela dépend de là où en est le groupe à ce moment-là de ses possibilités de mise en représentation.

\section{BIBLIOGRAPHIE}

Ancelin-Schutzenberger, A. 1970. Précis de psychodrame, Paris, Éditions Universitaires.

Anzieu, D. 1985. Le Moi-peau, Paris, Dunod.

Anzieu, D. 1987. «Les signifiants formels et le Moi-peau », dans D. Anzieu, D. Houzel et coll., Les enveloppes psychiques, Paris, Dunod, p. 1-22.

Anzieu, D. et coll. 1987. Les enveloppes psychiques, Paris, Dunod.

Aulagnier, P. 1975. La violence de l'interprétation, Paris, PuF.

Avron, O. 1996. La pensée scénique : groupe et psychodrame, Toulouse, érès.

BION, W.R. 1961. Recherche sur les petits groupes, Paris, PUF.

Bion, W.R. 1990. L'attention et l'interprétation, Paris, Payot.

Casement, P. 1985. À l'écoute du patient, Paris, PUF.

Foulkes, S.-H. 1964. Psychothérapie et analyse de groupe, Paris, Payot, 1970.

Gimenez, G. 1996. «La groupalité psychique dans la thérapie individuelle de schizophrènes ", Revue française de psychothérapie psychanalytique de groupe, $\mathrm{n}^{\circ} 27$, p. 109-119.

GimenEz, G. 2003. «Les objets de relation dans la thérapie individuelle et groupale de patients schizophrènes », Revue de psychothérapie psychanalytique de groupe, 41, p. 41-62.

FreUd, S. 1898. «Sur le mécanisme psychique de l'oubli », dans Résultats, idées, problèmes, I, Paris, PUF, 1984.

Freud, S. 1920. «Au-delà du principe de plaisir », dans Essais de psychanalyse, Paris, Payot, 1993, p. 41-111. 
FreUd, S. 1921. «Psychologie des foules et analyse du moi », dans Essais de psychanalyse, Paris, Payot, 1981, p. 117-217.

Freud, S. 1924. « Notice sur le bloc magique », Euvres complètes, XVII, Paris, PuF, 1992

Green, A. 1993. Le travail du négatif, Paris, Éditions de Minuit.

Houzel, D. 1994. «L'enveloppe psychique : métaphore et processus », dans R. Kaës et coll., Les voies de la psyché. Hommage à D. Anzieu, Paris, Dunod, p. 163172.

KAËs, R. 1982. Le travail psychanalytique dans les groupes, Paris, Dunod.

KAËs, R. 1993. Le groupe et le sujet du groupe, Paris, Dunod.

KAËs, R. 1994. La parole et le lien, Paris, Dunod.

KAËS, R. 1999. Les théories psychanalytiques du groupe, Paris, PUF.

KAËs, R. et coll. 1999. Le psychodrame psychanalytique de groupe, Paris, Dunod.

Le Goues, G. 1991. Le psychanalyste et le vieillard, Paris, PuF.

Le Goues, G. ; Peruchon, M. 1992. «Ultimes processus de pensée », Revue française de psychanalyse, $\mathrm{n}^{\circ} 1, \mathrm{p} .135-148$.

Ploton, L. 2009. «À propos de la maladie d'Alzheimer », Gérontologie et société, $\mathrm{n}^{\circ} 128-129$, p. $89-115$

Ploton, L. ; Cristini, C. 2009. " Mémoire et autobiographie », Gérontologie et société $\mathrm{n}^{\circ} 130, \mathrm{p} .75-95$.

Winnicott, D.W. 1971. Jeu et réalité, Paris, Gallimard. 